\title{
LONG-TERM CARE FACILITIES AND RESEARCH: HOW COVID-19 CHANGES THINGS
}

\author{
Instituições de longa permanência para idosos e a pesquisa: \\ as mudanças geradas pela COVID-19
}

Long-term care facilities (LTCF) provide 24-hour care predominantly for older people with complex physical or cognitive disabilities who can no longer be supported at home. They are configured differently and provide slightly different models of care between countries. ${ }^{1}$ Differences relate to funding models, how staff are trained, the balance between health and social care within organizational ethos, how healthcare inputs are co-ordinated, and how data are collated and used to assure and improve care. The long-term care sector differs in maturity between countries and is at its most developed in countries which are wealthier, and those with socialized healthcare systems. Regardless of these differences, the people cared for in LTCF are very similar internationally, and experience marked multimorbidity, frailty, and cognitive impairment. ${ }^{2}$ Many are nearing the end of their lives and, in most countries, older people who move into LTCF will subsequently die there. ${ }^{3}$

The differential maturity of long-term care sectors between countries is mirrored by differential development of research into LTCF worldwide. ${ }^{4}$ The literature shows that three stages have defined global research in this sector: an early stage (2000-2005), where studies primarily focused on care demand, functional, cognitive, and health status; a second stage (2006-2010), where the focus shifted to caregiving-related workforce factors; and a third stage (2011-2015), where attention moved to improving quality of care and to implementing clinical practice guidelines into LTCF. ${ }^{5}$ Global progress with research in long-term care has not been uniform, and many countries are still in the earlier stages of development. When we recently considered the maturity of research in Brazilian LTCF, we found that research there was still rooted very much in the first stage. ${ }^{6}$

Internationally COVID-19 has affected the residents of LTCF more adversely than any other population group. Mortality rates for COVID-19 in residents of LTCF have ranged from 0 to $66 \%$ of all COVID-related deaths. ${ }^{7}$ The reasons for the variation in mortality between countries are difficult to unpick. Variation is likely to be, in part, a consequence of the longstanding differences in how long-term care sectors are organized, as outlined above. But they will also relate to differences in national policies concerning COVID-19, particularly around: provision and availability of personal protective equipment; infection prevention and control procedures; utilization and integration of testing in LTCF; use of enhanced care for COVID19 in long-term care settings, and; vaccination policies, when they become available.

There is no doubt that, in most countries, LTCF have entered the public consciousness during the pandemic in a way that they have not done before. This has led to an unprecedented focus on the sector by policymakers, healthcare leaders, and research commissioners and funders.

From a policy perspective, we have seen solidification of guidelines and regulations around infection control, including recommendations for quarantine and isolation of older people with frailty in LTCF. ${ }^{8}$ Other initiatives have focussed on how enhanced therapies, such as oxygen and intravenous fluids, can be used in long-term care sectors in countries where they have not been deployed in the past, as part of a broader consideration of providing a better continuum of care for older people with frailty between the community in hospitals. ${ }^{9}$ In countries where routine monitoring of older people with acute illness in LTCF has not hitherto been routine, we have seen the introduction of sphygmomanometry, oximetry, and early warning scores. ${ }^{10}$ 
Following a swathe of early research describing the epidemiology of COVID-19 in LTCF, more recent projects have gone on to consider how interventions can improve care as the sector adapts to COVID-19. We have seen deployment point-of-care Reverse Transcriptase Polymerase Chain Reaction (RT-PCR) testing in LTCF, with evidence that they are feasible, can be integrated into workflows, and show good agreement with laboratory-based PCR, ${ }^{11}$ although such technologies are not so far widely deployed by comparison with much cheaper, but less accurate, lateral flow devices. ${ }^{12}$ Future research will consider the role of resident tracking technologies in outbreak control, ${ }^{13}$ the role of novel COVID19 therapies in LTCF, ${ }^{14}$ and pre and post-exposure COVID prophylaxis in the setting. ${ }^{15}$ The prevalent immunosenescence in the residents of LTCF means that further research about the impact of COVID-19 vaccines on outcomes for care recipients must be conducted. ${ }^{16}$

On the face of it, this rapid expansion of service delivery and research for a care sector, and section of the population, largely ignored in many countries in the past, is a source of celebration, a rare silver lining on the cloud which is COVID19. But nothing is without its risks. Researchers have played an important role in highlighting how frontline staff in LTCF, the people that know how care provision really works, have been largely excluded from planning many innovations implemented during the pandemic. ${ }^{17}$ At its best, this means that these innovations will need some future adaptation as we emerge from COVID-19. At its worst, it means that they are poorly conceived, impossible to implement, and need to be "worked around" by frontline staff. Academics have highlighted that frontline staff are historically under-recognized and underpaid, a situation that has not improved through the pressures and impositions of the pandemic. ${ }^{18}$
Researchers have also described the fragility of the data used to plan the COVID-19 response in some countries, with a call to arms for implementation of minimum datasets in all long-term care sectors internationally. ${ }^{19,20}$

A real risk in all of this is the rapid medicalization of LTCF. Quarantine, early warning scores, PCR assays, oxygen therapy, minimum datasets, and injectable immunologicals are the stuff of hospitals, not households. On the positive side, this will provide an evidence base that a greater spectrum of healthcare interventions in LTCF are feasible than hitherto considered. On the negative side, the opportunity costs associated with providing such care could lead to institutionalization and depersonalization of long-term care that must be resisted.

If ever there was a time for a rapid expansion of research into long-term care facilities, it is now. Regardless of how evolved long-term care sectors are, countries have an opportunity now to decide what shape they want to care for the most vulnerable people in society to take at the end of this pandemic. This will, and should, involve research to expand novel healthcare interventions and the infrastructure to support them to this population so that they can benefit from more care in situ. But it should also involve research into what residents, their families, and staff value, and research about the valuable contributions that residents, their families, and staff can make. This work is essential if we're to achieve a balanced approach that reflects the realities of care delivery on the ground.

Adam Lee Gordon

Professor of Care of Older People, University of Nottingham - Nottingham, United Kingdom. NIHR Applied Research Collaboration-East Midlands, Nottingham, United Kingdom.

\section{REFERENCES}

1. Schols J, Gordon A. Residential and nursing home care: from the past to the future. In: Michel JP, Beattie BL, Martin FC, Walston JD, editors. London: Oxford Textbook of Geriatric Medicine; 2017.

2. Achterberg WP, Everink IH, Van Der Steen JT, Gordon AL. We're all different and we're the same: The story of the European nursing home resident. Age Ageing. 2020;49(1):3-4. https://doi.org/10.1093/ ageing/afz145

3. Honinx E, Van Dop N, Smets T, Deliens L, Noortgate NVD, Froggatt $\mathrm{K}$, et al. Dying in long-term care facilities in Europe: The PACE epidemiological study of deceased residents in six countries. BMC Public Health. 2019;19. https://doi.org/10.1186/s12889-019-7532-4

4. Gordon A, Logan P, Jones R, Forrester-Paton C, Mamo JP, Gladman JRF. A systematic mapping review of Randomized Controlled Trials (RCTs) in care homes. BMC Geriatr. 2012;12:31. https://doi. org/10.1186\%2F1471-2318-12-31

5. Fu L, Sun Z, He L, Liu F, Jing X. Global long-term care research: A scientometric review. Int J Environ Res Public Health. 2019;16(12):2077. https://doi.org/10.3390/ijerph16122077

6. Jacinto A, Achterberg W, Wachholz P, Dening T, Dening H, Devi R, et al. Using International Collaborations to Shape Research and
Innovation into Care Homes in Brazil: A White Paper. J Nurs Home Res. 2020;6:109-13. https://doi.org/10.14283/jnhrs.2020.28

7. Comas-Herrera A, Zalakaín J, Lemmon E, Herdenson D, Litwin C, Hsu AT, et al. Mortality associated with COVID-19 outbreaks in care homes: early international evidence [Internet]. LTC Responses to COVID-19; 2020 [cited on 2021 Jan 9]. Available at: https://tccovid. org/2020/04/12/mortality-associated-with-covid-19-outbreaks-incare-homes-early-international-evidence/

8. Blain H, Rolland Y, Schols JMGA, Cherubini A, Miot S, O'Neill D, et al. August 2020 Interim EuGMS guidance to prepare European LongTerm Care Facilities for COVID-19. Eur Geriatr Med. 2020;11:899913. https://doi.org/10.1007/s41999-020-00405-z

9. British Geriatrics Society. Managing the COVID-19 pandemic in care homes for older people [Internet]. British Geriatrics Society; 2020 [cited on 2020 Nov 23]. Available at: https://www.bgs.org.uk/ resources/covid-19-managing-the-covid-19-pandemic-in-care-homes

10. Wessex Academic Health Sciences Network. COVID Oximetry at Home Toolkit [Internet]. Wessex Academic Health Sciences Network [cited on 2021 Jan 5]. Available at https://wessexahsn.org.uk/ projects/388/covid-oximetry-at-home-toolkit 
11. Micocci M, Gordon AL, Seo MK, Allen AJ, Davies K, Lasserson $D$, et al. Is Point-of-Care testing feasible and safe in care homes in England? An exploratory usability and accuracy evaluation of Point-of-Care Polymerase Chain Reaction test for SARS-COV-2. medRxiv. 2020;2020.11.30.20240010. https://doi. org/10.1101/2020.11.30.20240010

12. Deeks JJ, Raffle AE. Lateral flow tests cannot rule out SARS-CoV-2 infection. BMJ. 2020;371. https://doi.org/10.1136/bmj.m4787

13. University of Leeds. Can wearable technology help care homes save lives [Internet]. University of Leeds [cited on 2021 Jan 5]. Available at: https://www.leeds.ac.uk/news/article/4686/can_wearable_ technology_help_care_homes_save_lives

14. Mahase E. Covid-19: Anti-TNF drug adalimumab to be trialled for patients in the community. BMJ. 2020;371:m3847. https://doi. org/10.1136/bmj.m3847

15. National Institute of Health Research. PROphylactic TrEatment of COVID in Care Homes Trial (PROTECT) [Internet]. National Institute of Health Research [cited on 2021 Jan 5]. Available at: https:// fundingawards.nihr.ac.uk/award/NIHR133443
16. Cox LS, Bellantuono I, Lord JM, Sapey E, Manick JB, Partridge L, et al Tackling immunosenescence to improve COVID-19 outcomes and vaccine response in older adults. Lancet Heal Longev. 2020;1 (2):e55-7. https://doi.org/10.1016/s2666-7568(20)30011-8

17. Marshall F, Gordon AL, Gladman JR, Bishop S. Care homes, their communities, and resilience in the face of the COVID-19 pandemic interim findings from a qualitative study. medRxiv. 2020;2020.11.10.20229013. https://doi.org/10.1101/2020.11.10.20229013

18. McGilton K, Escrig-Pinol A, Gordon A, Chu CH, Zúñiga F, Sanchez MG et al. Uncovering the Devaluation of Nursing Home Staff During COVID-19: Are We Fuelling the Next Health Care Crisis? J Am Med Dir Assoc. 2020;21(7):962-5. https://doi.org/10.1016/j.jamda.2020.06.010

19. Hanratty B, Burton JK, Goodman C, Gordon AL, Spilsbury K. Covid-19 and lack of linked datasets for care homes. BMJ. 2020;369:m2463. https://doi.org/10.1136/bmj.m2463

20. Burton JK, Goodman C, Guthrie B, Gordon AL, Hanratty B, Quinn TJ. Closing the UK care home data gap - methodological challenges and solutions. Int J Popul Data Sci. 2020;5(4):3. https://doi.org/10.23889/ ijpds.v5i4.1391 